Available online at www.eccomasproceedia.org

Eccomas Proceedia UNCECOMP (2021) 232-245

ECCOMAS

Proceedia
UNCECOMP 2021

$4^{\text {th }}$ ECCOMAS Thematic Conference on Uncertainty Quantification in Computational Sciences and Engineering M. Papadrakakis, V. Papadopoulos, G. Stefanou (eds.) Streamed from Athens, Greece, 28 -30 June 2021

\title{
ANALYTICAL MODEL FOR FRACTURE IN RANDOM QUASIBRITTLE MEDIA BASED ON EXTREMES OF THE AVERAGING PROCESS
}

\author{
Miroslav Vořechovskś ${ }^{1}$ \\ ${ }^{1}$ Brno University of Technology \\ Veveří 95, Brno, 602 00, Czech Republic \\ e-mail: vorechovsky.m@vut.cz
}

\begin{abstract}
The paper presents an analytical model for prediction of the peak force in concrete specimens loaded in bending (both notched and unnotched). The model is capable of predicting peak force statistics by computing the extreme values of sliding averages of random strength fields. The local strength of the specimen is modeled by a stationary isotropic random field with Gaussian distribution and a given autocorrelation function. The averaging operation represents the progressive loss in material integrity and the associated stress redistribution that takes place prior to reaching the peak load. Once the (linear) averaging process is performed analytically, the resulting random field of averaged strength is assumed to represent a series of representative volume elements (RVEs) and the global strength is found by solving for the minimum of such an effective strength field. All these operations can be written analytically and there are only four model parameters: the three dimensions of the averaging volume (RVE) and the length of the final weakest-link chain. The model is verified using detailed numerical computations of notched and unnotched concrete beams simulated by mesoscale discrete simulations of concrete fracture performed with probabilistic distributions of model parameters. The numerical model used for verification represents material randomness both by assigning random locations to the largest aggregates and by simulating random fluctuations of material parameters via a homogeneous random field.
\end{abstract}

Keywords: quasibrittle structure, concrete, discrete model, fracture process zone, random strength field, local averaging, weakest-link model. 


\section{INTRODUCTION}

The mechanical responses of heterogeneous quasibrittle materials like concrete are intrinsically intertwined with non-homogeneity and randomness at finer scales of material resolution. In order to represent the cascading events of crack nucleation, growth and their interaction a variety of models have been developed.

Taking into account the spatial variation of material properties is also of paramount importance in the safety and reliability evaluation of engineering structures. Nowadays, the simplest and most oft-used method to account for material spatial randomness is classical Weibull theory $[21,20]$. The structure is viewed as composed of many small reference volumes that are independent and have random strengths, no redistribution of stresses is considered, and the failure of any piece of material triggers the failure of the whole system. This holds even for nonuniform stress fields: the Weibull theory allows to compute an integral over the structure volume the result of which can be interpreted as the equivalent number of equally stressed material elements. The input to this Weibull integral is the shape of the stress field just before failure; see e.g. Sec. 4.1 in [19] for details. In this approach, all information about the mechanics of failure is lost, and the structural geometry becomes irrelevant: the integral transforms the structure into uniaxial bar with a constant stress. Indeed, from the viewpoint of Weibull theory, any piece of material can be viewed as a chain of elements (in series coupling) and thus statically determinate. This is unrealistic for real materials. Another caveat relates to the assumption of the spatial independence of local strengths. Weibull distribution is one of the three stable forms of extreme value distributions [10]. Its derivation assumes the survival probability of the structure as the product of survival probabilities of all infinitesimal pieces of material, which is only correct if these survival probabilities are independent. Spatial correlation incorporates a length scale, though, and the Weibull theory must be modified accordingly [17]. There is also a difficulty associated with the correct determination of the effective number of dimensions, as not only the geometrical similarity influences it. If, for example, a 3D structure fails only after the whole thickness fails (no matter how much the thickness is), the 3D geometrical scaling represents 2D scaling in Weibull theory, which is sometimes a source of confusion in experimental data interpretation; see [19] or Sec. 12.3.3 in [2]. Another example is when the failure can be triggered by two different and independent failure mechanisms: either a piece of material fails in the volume or by failure triggered at surface flaw. This is possible in the theory and as the structure is scaled in 3D, the amount of volume scales with the third power but the amount of surface material is scaled quadratically. Another problem with a direct application of Weibull theory based on the elastic stress field is that in cracked bodies or bodies with sharp notches, the singularity of the stress field causes the Weibull integral to diverge, thus predicting an infinite effective volume and zero strength. The self-similarity embodied in classical Weibull theory means that the strength of any piece of material is Weibullian, and only the volume and effective dimensionality decide the scale parameter; the shape parameter is size-invariant. These assumptions are not acceptable, and an alternative model must be developed to predict statistical strength in a manner that reflects the true behavior of heterogeneous materials such as concrete.

In this paper, we use results obtained with a particular class of discrete mesoscale models $[5,6]$ which was enhanced [9] by additional spatial variability in material properties via random fields. The discrete mesoscale models exhibit a certain variability in response due to the random placement of numerical aggregates which leads to random dimensions and orientations of "bars" connecting the aggregate centers. These random geometrical properties, when combined with deterministic materials properties, lead to scatter in structural response such as the peak 
loads, sequences of local events and the related crack trajectories etc. Studies performed in [9] showed that scatter is not representing the experimental data obtained with concrete and therefore an additional sources of randomness was introduced. Random fields of material parameters provide spatial variability in a controlled fashion. Not only that the distribution of local properties can be described by the distribution of a random field, but also the spatial correlation is under control. It has been found that there exist a meaningful set of parameters of random fields used for modeling the otherwise deterministic parameters in the discrete model to accurately mimic the experimental data [9]. However, the connection of these spatial variability parameters to material and specimen production is not completely clear. Therefore, one can assume a range of possible setting of random fields to obtain a range of possible structural responses when predicting the true behavior.

A systematic study regarding the effect of parameters of the random fields has been performed in [8] to show the effect on both notched and unnotched structural elements made of concrete. It was shown that notched structures with stress concentration are affected by statistical variability in a different way than unnotched ones and that behavior of concrete bars under pure tension may behave very differently depending on a particular realization of the local strength field. The present paper, which is a promotion of a recent journal paper [18] provides a simple explanation to the different behavior of notched and unnotched specimens and proposes a simple analytical model that can replace the expensive random discrete mesoscale simulations. The analytical model is able to predict the peak force statistics by computing the extreme values of sliding averages of random strength fields.

\section{OVERVIEW OF THE PROPOSED ANALYTICAL MODEL}

The Introduction section discussed the classical Weibull theory along with a critique of its weaknesses. Several assumptions were found unacceptable and therefore an alternative model for the prediction of statistical strength is developed here so as to better reflect the true behavior of heterogeneous concrete-like material. We formulate the following simple hypotheses embodied in the proposed analytical model:

1. There is a representative volume element (RVE) that can be identified for a given material, geometry, dimensions, boundary conditions, etc., which is defined in such a manner that its failure leads to the exhaustion of structural strength (peak load). The dimensions of the RVE are not dependent on the parameters of the local strength random field. The volume of structure can be discretized into many RVE subvolumes.

2. Each RVE is composed of many microbonds that contribute to its strength. Within each RVE, one can define effective strength as the moving average (within RVE volume) of local strengths found within that RVE. This RVE strength is random and is a result of combinations of both serial and parallel couplings of these microbonds. The inside of each RVE can potentially undergo stress redistribution in response to a change in the external loading. Depending on the redistribution potential of the material, the RVE can have different degrees of brittleness/ductility and the probabilistic distribution of its strength is influenced correspondingly. Effective strength may be a random function and it represents a barrier for the effective stress (action) defined in the next item.

3. During loading, one can define effective stress at each potential RVE center as the local average of stresses found within that RVE. Thus effective stress is a homogenized part of stress obtained as an average over the RVE volume defined in item 1. The effective stress evolves during the loading process.

4. The structure can be discretized into many potential RVEs that may or may not share 

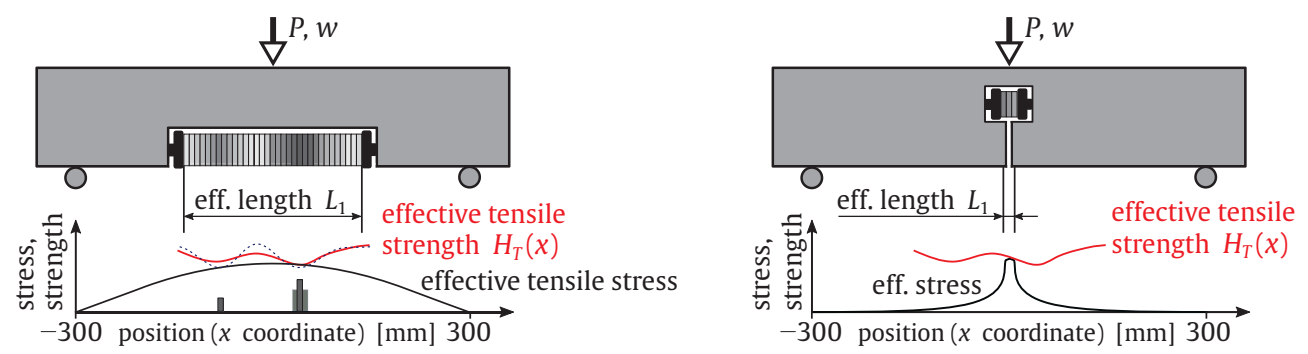

Figure 1: Illustration of the transformation of the random 3D discrete mesoscale model into an effective 1D model of a chain of random RVEs. From the left: unnotched bending, notched bending and uniaxial tension.

an identical level of load expressed via the effective stress. We assume that at the moment of attaining the peak load of the structure, the inelastic strains localize into a single macrocrack inside one of the RVEs. At this moment the effective (tensile) strength of that critical RVE attains its effective stress. This moment corresponds to the structural failure because the exhaustion of the effective tensile strength of a critical RVE signals the peak load of the whole structure.

5. The failure of any RVE occurs after redistribution of stresses takes place inside the RVE volume. The failure typically occurs after many of the bonds within the RVE exceed their random capacity. The number of these bonds increases with the redistribution potential expressed via toughness or effective fracture energy. Even though the model features a strength-based failure criterion, fracture energy influences the dimensions of the RVE. The effective strength of a potential RVE is effectively Gaussian within a wide range around the mean strength; the degree of normality (spread of the Gaussian core) increases with the number of parallel couplings involved in the averaging operation (and thus with the RVE size). In concrete, the strength probability distribution function can be reasonably considered Gaussian in the central region. This distribution can be considered to have gradual transitions towards power law tails $[7,11,1,17]$.

Item 1 defines the RVE in a different way than is usual in the modeling of heterogeneous materials. We remark that the term 'representative volume element' may have various meanings $[12,13,14,15]$. The problem with the definition of an RVE is that it depends on the property is to be represented [4], and therefore its size depends on the type of treated physical phenomena, microstructure geometry and the contrast between microstructure constituents [16].

Moreover, the effective strengths of a potential RVE can generally be statistically dependent and we assume that the effective strength is an autocorrelated random field, which is not considered in applications of the extreme value theory to the weakest-link model of independent links. The random field of effective strength is a result of the redistribution of stresses within an RVE volume/window, and the redistribution is taken into account simply via the moving average operation. Therefore, the above-mentioned weaknesses of the classical Weibull theory are removed: stress redistribution is taken into account (it incorporates a length scale) and so is the spatial correlation of RVE volumes (it incorporates another length scale, the effective autocorrelation length, which depends on both the autocorrelation of local strengths of a finer scale and the RVE window size). Another deviation from the Weibull theory is that the distribution of effective strength is no longer Weibullian (with the size-independent shape and scale parameter being scaled from a reference one corresponding to a reference size). 


\section{APPLICATION TO RANDOMIZED MESOSCALE DISCRETE MODEL}

Consider a unit-mean Gaussian random field $h(\boldsymbol{x})$ where the spatial coordinate $\boldsymbol{x}$ is defined in three dimensions (the beam volume). This random field represents the local strength (or simply its dimensionless multiplier as in the current application). It is a random function that depends on the spatial coordinate, $\boldsymbol{x}$. We consider $h$ to be a homogeneous random field in the strong sense, meaning that the distribution of the random field is independent of the location, and that the autocorrelation structure among the local random variables is also shift-invariant, i.e. it only depends on the mutual distance between the points. We also consider that $h(\boldsymbol{x})$ is ergodic (the spatial average is equal to the ensemble average). Thus, our random field is fully defined by the constant mean value $\mu_{h}=1$, standard deviation $\delta_{h}$ and autocorrelation function $\rho\left(\tau ; \ell_{\rho}\right)$, where $\|\boldsymbol{\tau}\|$ is the lag (distance between two spatial points). This function is considered to be a separable isotropic Gaussian (squared-exponential) autocorrelation function

$$
\rho\left(\boldsymbol{\tau} ; \ell_{\rho}\right)=\exp \left[-\left(\frac{\|\boldsymbol{\tau}\|}{\ell_{\rho}}\right)^{2}\right]=\prod_{v=1}^{3} \exp \left[\left(-\frac{\tau_{v}}{\ell_{\rho}}\right)^{2}\right], \quad\left|\tau_{v}\right| \geq 0
$$

The separability means that the correlation between two different random variables $h\left(\boldsymbol{x}_{1}\right)$ and $h\left(\boldsymbol{x}_{2}\right)$ is a product of autocorrelations that depend solely on distances $\tau_{v}$, i.e. projections of the lag $\|\boldsymbol{\tau}\|=\sqrt{\sum_{v}^{3} \tau_{v}^{2}}$ along individual dimensions $v=1,2,3$. Therefore, for such a fully separable autocorrelation, we can write

$$
\rho(\boldsymbol{\tau})=\rho\left(\tau_{1}, \tau_{2}, \tau_{3}\right)=\rho\left(\tau_{1}\right) \rho\left(\tau_{2}\right) \rho\left(\tau_{3}\right)
$$

The isotropy means that the autocorrelation length, $\ell_{\rho}$, is identical in all three directions.

The additional randomness due to the spatial variability of material properties in the discrete model is incorporated into the constitutive relation via modifying the main parameters that control the tensile response of the material unsing a random field $h(\boldsymbol{x})$. These parameters, which are associated with the individual contacts/bonds of the discrete model, are the tensile strength and fracture energy $f_{\mathrm{t}}$ and $G_{\mathrm{t}}$. As discussed in [8], the parameters are randomized via only one random spatially varying multiplier $h(\boldsymbol{x})$ in such a way that the local Irwin/Hillerborg characteristic length is kept unmodified and constant throughout the beam volume. This is achieved [19] by taking $f_{\mathrm{t}}(\boldsymbol{x})=\bar{f}_{\mathrm{t}} h(\boldsymbol{x})$ and $G_{\mathrm{t}}(\boldsymbol{x})=\bar{G}_{\mathrm{t}}[h(\boldsymbol{x})]^{2}$, where $\bar{f}_{\mathrm{t}}$ and $\bar{G}_{\mathrm{t}}$ denote the deterministic model parameters. If $h$ is a constant (independent of $\boldsymbol{x}$ ), two beam simulations that differ only in two values of a random multiplier, say $h_{1}$ and $h_{2}=c \cdot h_{1}, c>0$, will follow exactly the same cracking process and the computed forces will simply have a ratio of $c$.

The probabilistic part of the model is fully characterized by a unit-mean Gaussian homogeneous random field $h(\boldsymbol{x})$ which has two free parameters: the coefficient of variation, $\delta_{h}$, and the autocorrelation length, $\ell_{\rho}$, which is the parameter of the selected squared-exponential isotropic autocorrelation function. The random field is stationary in the strong sense, meaning that the distribution is identical throughout the whole domain (specimen volume), and also the autocorrelation structure is shift-invariant. In fact, the distribution function of the strength multiplier, $h(\boldsymbol{x})$, should be considered to have a modified left tail - it was assumed to follow Weibull-Gaussian distribution, i.e. Gauss distribution with a Weibullian left tail grafted at very low probability, see [8]. The cumulative distribution function of Weibullian random variable $h$ reads $F_{W}(h)=1-\exp \left[-(h / s)^{m}\right]$ where $m$ and $s$ are the shape and scale parameters, respectively. The left tail grafted at very small values of strength practically does not influence the analysis performed here with purely Gaussian distribution. 

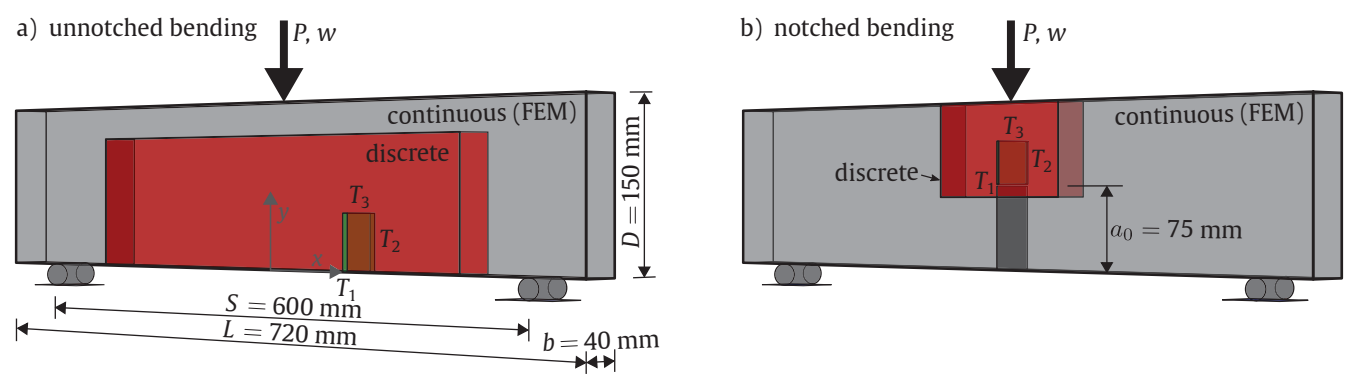

Figure 2: Specimen geometry for a) unnotched and b) notched beams loaded in three point bending. The narrow blocks of dimensions $T_{1}, T_{2}$ and $T_{3}$ are the three RVE length parameters of the proposed analytical model.
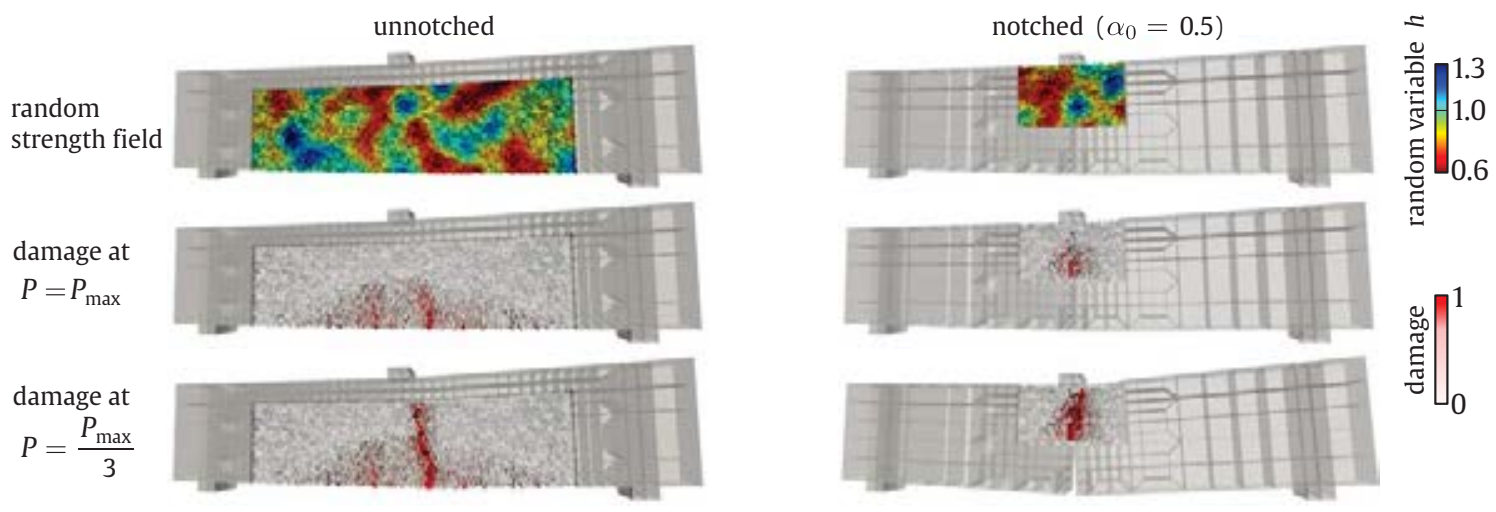

Figure 3: Distribution of damage at the inter-particle contacts of one realization of the probabilistic model $\left(\ell_{\rho}=\right.$ $25 \mathrm{~mm}$ ) at the peak load (top) and at the termination of the simulation (bottom) of unnotched and notched beams. For definition of "damage", please see the description of the mesoscale discrete model in [8].

The random fields used to represent material parameters are generated with various autocorrelation lengths spanning from $\ell_{\rho} \rightarrow 0$ (independently sampled random variables) up to the infinitely long autocorrelation length $\ell_{\rho} \rightarrow \infty$, for which the realizations are random constant functions, and therefore the whole structure shares the same value in a single realization. The strengths of the beams were statistically evaluated as functions of the autocorrelation length and variance of the random field.

The model was employed to simulate the three point bending of concrete beams with and without a central notch, see Fig. 2. Fig. 3 presents examples of the typical patterns of damage for selected realizations of the random strength field multiplier. One can see that the damage is more localized in the notched beams.

In the two bending geometries considered here (unnotched and notched three point bend beams), the identification of the "chain of RVEs" is particularly simple. By studying the stress fields of the beams in the discrete model at the peak load, we can compute the effective stresses at the peak load. Fig. 1 illustrates the idealization used in the analytical model. The failure of the weakest RVE thus corresponds to the flexural strength (peak load) of the beam. Each of such RVEs is assumed to be a cuboid; see the thin green cuboids in Fig. 2. The series coupling of potential RVEs over the effective length is depicted in the highly tensioned zones. At the bottom, the averaged stress fields and a realization of the effective strength field are depicted together with the decisive macrocrack location. Thanks to the ability to redistribute stress internally, the tensile stress fields can be considered roughly constant over a certain length at the bottom part of the beams. To support this claim, the effective tensile stresses obtained from one realization of the beams modeled by the discrete mesoscale simulations are depicted 

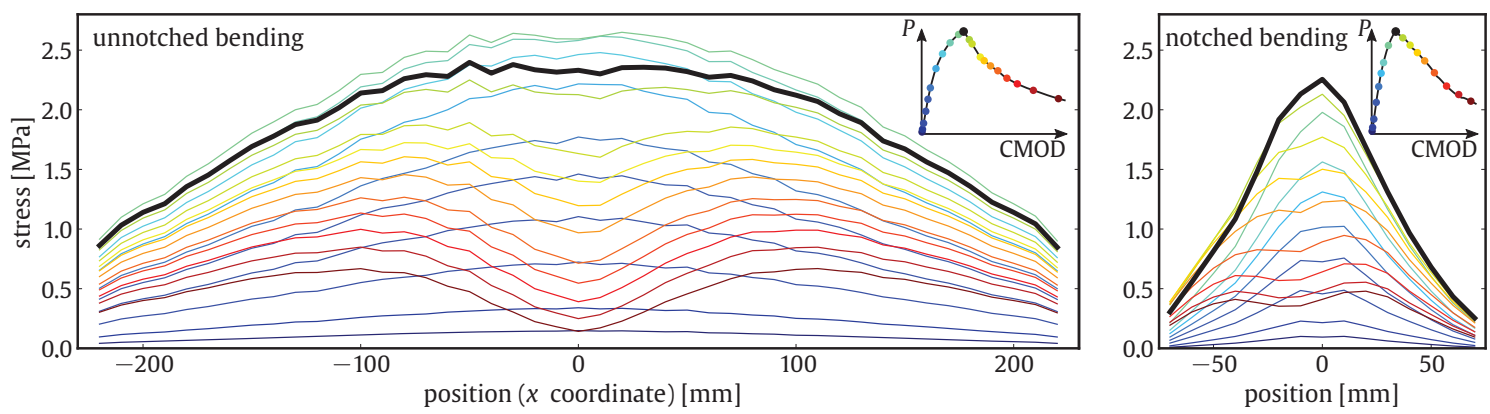

Figure 4: Evolution of the effective stress in three point bend beams as predicted by the discrete mesoscale model. The line colors correspond to various stages of loading: pre-peak stages (thin blue), peak load (thick black), right after peak load (thick green) and postpeak stages (red to brown); [18].

in Fig. 4. These profiles of the effective tensile stresses are calculated as sliding averages of windows corresponding to the RVE sizes (length about $10 \mathrm{~mm}$, depth $40 \mathrm{~mm}$ or $50 \mathrm{~mm}$ and beam thickness $40 \mathrm{~mm}$ ), see below. In the case of unnotched beams, the length of an effective chain can become as great as $300 \mathrm{~mm}$, which is one half of the bending span. In the notched beams, however, the length is very limited as the effective stress field is very localized around the notch tip. Note that the effective stress profile does not have to be constant and more complicated forms can also be considered when computing this effective chain length. The effective stress field (action) is considered approximately deterministic from here on. It is true that the averaged stress exhibits a certain degree of variability influenced mainly by the size of the averaging window. Indeed, various realizations of the mesoscale model (different positions of the grains) return slightly different the stress fields. However, the averaging window contains many grains and therefore the variance in the effective stress can be disregarded; see Fig. 4. A rigorous approach to incorporate this variability has been presented in [22].

The most important information gained from the analyses [8] performed with the probabilistic mesoscale model is that the volume within which massive redistribution takes place right at the peak load is almost independent of the parameters of the random field. As shown in [18], when considering the strengths of individual bonds being described by a random field, the effective strength parameters of each RVE (an averaging window) can be predicted analytically, together with the spatial correlation of these averaged strengths. Therefore, the three-dimensional problem is transformed into a one-dimensional problem (a chain) with an effective strength variable along the beam span, see Fig. 1. The effective strength becomes a random process that can be mathematically described as a result of the moving average of a local random strength field [18].

When this effective strength profile is being attained by a constant effective stress, it suffices to merely obtain the minimum of a random field over a certain effective length [18].

Since local strengths at the mesolevel are almost entirely Gaussian, the effective strength of individual potential RVEs is Gaussian, too. This is due to the averaging operation that suppresses the tails (by virtue of the central limit theorem the Gaussian core spreads wider). Therefore, it suffices to focus on the extremes of Gaussian random fields over a closed interval: the effective length $L_{1}$. This length must be determined by considering both the stress field and the properties of the sample paths of the effective strength (its gradients). In the studied examples of three point bending and tension, the estimation of chain length is provided in [18]. 


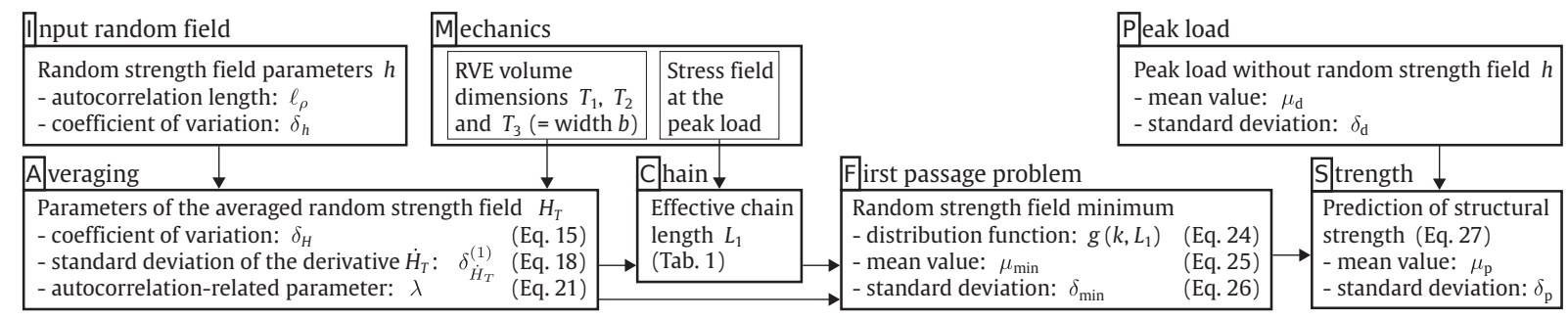

Figure 5: Flow chart of the model. The top row represents the input information for the proposed model sketched in the bottom row. Equation numbers correspond to equations in [18].

\subsection{Three point bend beams}

The application of the proposed model to bending requires either (i) an analysis of the exceedance probability function that features non-constant effective threshold (stress) $u(x)$ over the whole bending span, or (ii) determination of the effective length of chain in which the macrocracks appear and in which the effective stress can be considered approximately constant. The first alternative is generally possible, but it leads to a somewhat more complicated formula for the probability density function of exceedance.

In this work we have selected the assumption of a roughly constant effective stress profile by taking advantage of the fact that there is an effective chain length $L_{1}$ which covers the range of high stresses where a crack occurs and in which the effective stress does not vary considerably.

The flow of the information in the proposed model is sketched in flowchart in Fig. 5. The information about random strength field (dimensionless local strength multiplier; see box I) is processed analytically to represent averaging using the estimated RVE size (the three dimensions of RVE can be estimated or identified from a detailed model for the mechanics; see box $M)$. The result of the averaging operation is a detailed description of the transformed effective strength random field $(A)$. Another information needed that represents the mechanics is the stress field at the peak load (M); in particular, we need to estimate the extent of regions that share almost identical effective stress at the peak load. Using this information and the data from effective strength random field, the effective "chain length" can be deduced (C). The next step is simply computation of the minimum strength over the effective length $(F)$. In cases when there is no other source of randomness, the computed mean value and standard deviation can be readily used as multipliers of the deterministic peak load. In our case, there is another source of variability in the peak load predicted by the discrete mesoscale model, see below. This source is not controlled by the random strength field and therefore this information $(P)$ is additionally passed to obtain the final result: the prediction of random structural strength parameters $(\mathrm{S})$; see also Sec. 3.3 on this topic. Note that box $(P)$ can also be used to carry information about additional sources of randomness independent from local strength random field; e.g. due to testing imperfections etc.

\subsection{Identification of the model parameters}

There are in total four parameters of the proposed analytical model that must be inferred (i.e. identified) from the discrete simulations: $T_{1}, T_{2}, T_{3}$ and $L_{1}$; see box $\mathrm{M}$ in Fig. 5. These are (i) the three dimensions of the local averaging RVE window (a volume whose failure triggers the failure of the whole structure), and (ii) the length of the effective chain (plus the information about the effective stress function over the length of this chain). The rectangular cubes of the RVE are assumed to have identical dimensions: the length $T_{1}$ measured along the beam span, the depth $T_{2}$ measured along the vertical axis, and the width $T_{3}$; see the illustration of one such 
RVE in Fig. 2. With these parameters, the probability distribution function of structural strength is obtainable analytically [18].

We first focus on the three dimensions of the averaging RVE volume. The width $T_{3}$ is not a free parameter: it must be taken as the beam width, $b=40 \mathrm{~mm}$, through which the crack front must pass. The lengths $T_{1}$ and $T_{2}$ are two length parameters that must be obtained either from a nonlinear analysis (e.g. with a discrete model) or estimated. As argued in, e.g. [3, 2], in the case of modulus of rupture (unnotched beams) there is a boundary layer of microcracking that develops prior to reaching the peak load. The depth of this layer, $D_{b}$, is approximately proportional to the maximum aggregate size, and thus independent of the structural size. We use the averaging depth $T_{2} \approx 2 D_{b}$, which is based on simulations performed in [8]. Indeed, the lengths $T_{1}$ and $T_{2}$ represent the width and depth of the region surrounding the macrocrack that forms at the peak load, or right after it has been reached: see Fig. 7 in [8]. Numerous analyses with the discrete model have confirmed that these lengths are not considerably dependent on the parameters of the random field; they can be obtained from the "deterministic" model in [8]. The depths $T_{2}$ of RVEs in the case of unnotched and notched beams do not differ much [18].

The averaging dimension $T_{1}$ is controlled by the irregular inner structure and also by the macroscopic stress field. In the direction of $x_{1}$, the averaging width $T_{1}$ is considered because it is known that the crack, once it has localized, is not perfectly planar. The width $T_{1}$ is related to the maximum aggregate size, $d_{\max }=10 \mathrm{~mm}$, and we conjecture that the fracture energy and the stress field have additional influence: in the case of unnotched beams, we have found that the crack "planes" are somewhat tortuous and therefore the RVE width $T_{1}$ is greater than in the case of notched beams that are exposed to strongly localized stress fields; see Fig. 4. Note that the relatively large averaging lengths $T_{2}$ and $T_{3}$ make the strengths within each RVE almost constant over directions $x_{2}$ and $x_{3}$. The only variability in space takes place along direction $x_{1}$, and this is what allows us to perform the one-dimensional idealization into the "effective chain" of RVEs. The RVE dimensions are thus selected.

The last, fourth, parameter of the model is the effective length $L_{1}$. It is the extent of the zone within which cracks will frequently appear in the beam, and is therefore dependent on the effective stress field (obtained by averaging with the three RVE dimensions). In both types of beams, however, the length $L_{1}$ is also influenced by the random field of the effective strength. This is because both the autocorrelation length and the variance influence the random gradient of the wavy function describing the RVE strengths along the beam. The effective chain has the length of the interval where the effective stress may attain the effective strength, and therefore it depends on both processes, see [18] for more details.

The unnotched beams have, at the peak load, a very long zone of almost constant stress leading to large $L_{1}$. These mild functions develop thanks to the redistribution capacity of the material. The shape of the effective stress (action) is parabolic; see Fig. 4. Therefore, its gradient is an almost exactly linear decreasing function. The gradient of the effective strength (barier) is a Gaussian random variable with zero mean and standard deviation $\delta_{\dot{H}_{T}}$. The length over which the two processes can meet is therefore proportional to $\delta_{\dot{H}_{T}}$ (with the dimension of load per distance) and inversely proportional to the slope of the decreasing first derivative of the stress process (i.e. inversely proportional to its constant curvature). The situation is illustrated in Fig. 6. By displaying several realizations of the averaged random field, one can see how the strengths compare to the same effective stress profile for various variances and autocorrelation lengths of the local strength.

In the case of notched beams, the effective stress field is very localized and the length $L_{1}$ is very short. The crack must initiate at the notch tip, but various cracks form a "fan" depending 


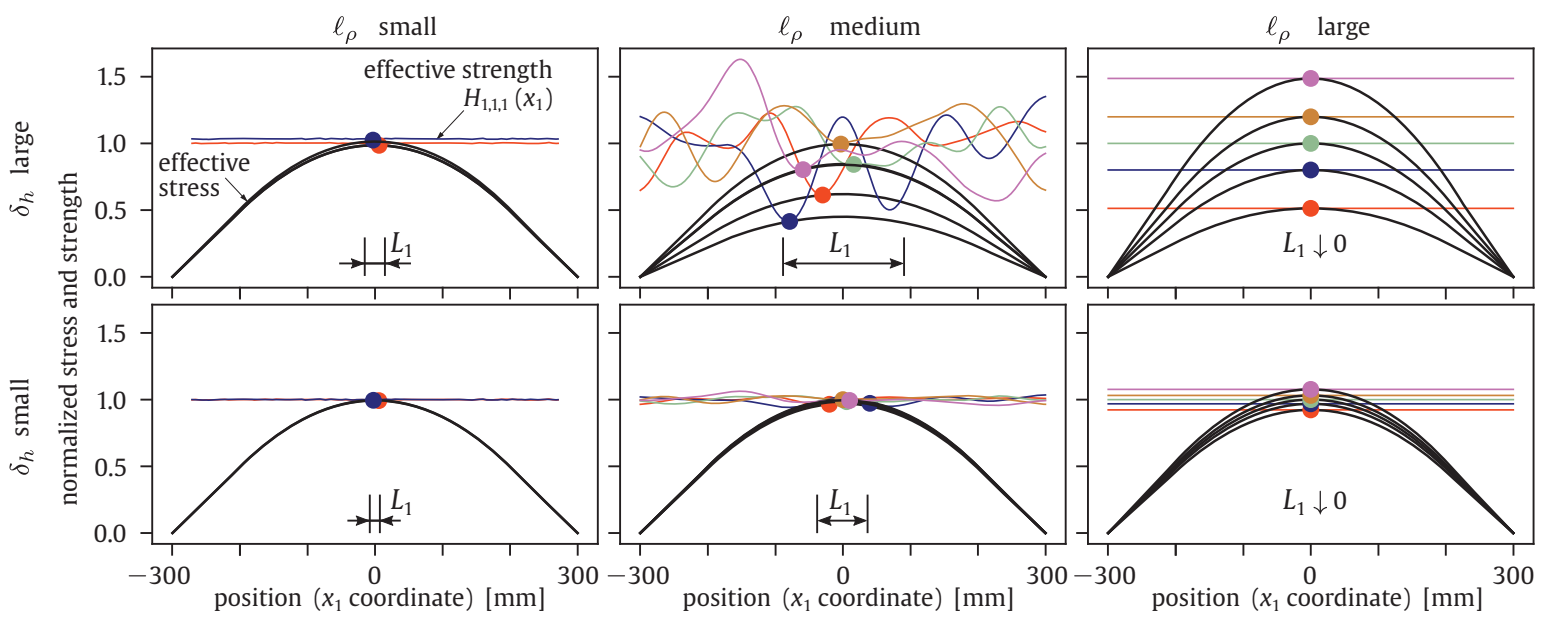

Figure 6: Illustration of the dependence of effective span $L_{1}$ (chain length) on the autocorrelation length $\ell_{\rho}$ and variance $\delta_{h}^{2}$ of the local (non-averaged) random field, [18]. The effective stress field is depicted for the unnotched beams. Top row: low variance. Bottom row: large variance.

on the random barrier ahead of the current crack tip.

\subsection{Comparison with the discrete mesocale model}

Having fixed all the parameters of the proposed analytical model, one could now compare the model with results of the parametric study presented in [8]. Fig. 7 presents a comparison between (i) the sets of the random discrete mesoscale simulations (a circle with errorbars representing $\mu_{\mathrm{p}} \pm \delta_{\mathrm{p}}$ ) and (ii) the results obtained by the proposed model (a solid line surrounded by a scatterband). An excellent agreement between the analytical predictions and the random discrete mesoscale simulations is obtained for the whole studied range of parameters $\ell_{\rho}$ and $\delta_{h}$ of the local random strength field.

We first comment on the asymptotic behavior, i.e. when the autocorrelation length approaches either zero or infinity. When $\ell_{\rho} \downarrow 0$, the local averaging within an RVE effectively removes any variability in the effective random strength field $\left(\delta_{H}=0\right)$. Therefore, the chain strength has zero variance, $\delta_{\min }=0$ and $\mu_{\min }=1$. Thus, the model predicts that the deterministic model solely governs the behavior

$$
\mu_{\mathrm{p}, 0}=\mu_{\mathrm{d}}, \quad \delta_{\mathrm{p}, 0}=\delta_{d}
$$

The results obtained from the random discrete mesoscale model slightly differs for two reasons: (i) in the random discrete model, there is still room for a limited weakest-link principle, and the separation of randomness in the "deterministic model" and the local random strength field is only an approximation; and (ii) the finite size of contacts/particles does not allow $\ell_{\rho}$ to decrease below the model resolution. Indeed, especially when the random field variability is high $\left(\delta_{h}=\right.$ 0.28), the variability of local strengths is not averaged out completely within the FPZ. However, the differences between the predictions of the discrete model and the analytical model are minor.

On the other extreme, when $\ell_{\rho} \uparrow \infty$, the realizations of effective strength processes along the chain are random constant functions with a unit mean value and a standard deviation that is not affected by the averaging: $\delta_{H}=\delta_{h}$. Since the chain length $L_{1}=0$, the standard deviation of the weakest RVE is $\delta_{\min }=\delta_{h}$ and the mean value remains $\mu_{\min }=1$. Therefore, the structural strength is simply the multiple of two independent sources of variability and the mean value 

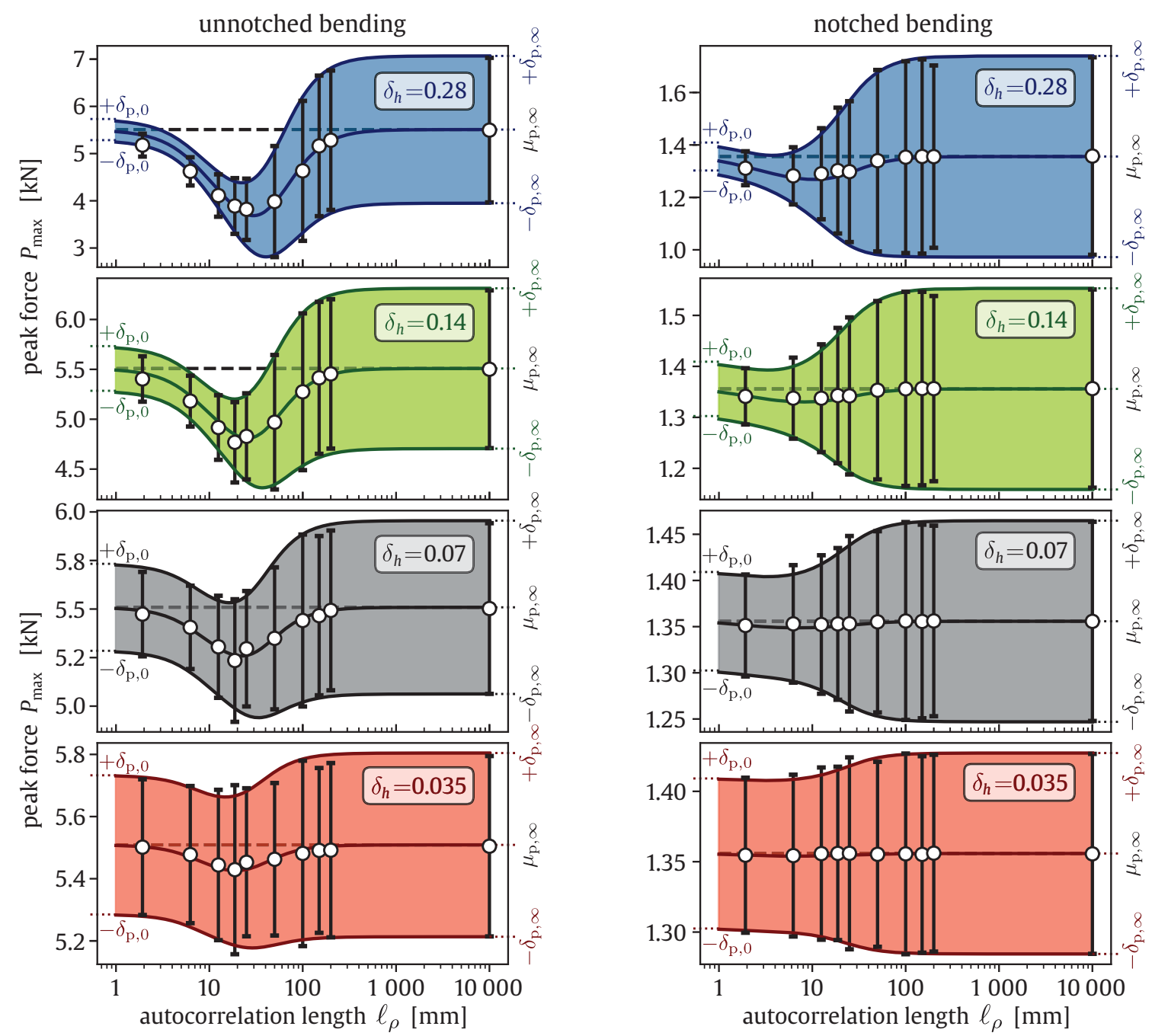

Figure 7: The mean value and standard deviation of the maximum load computed on unnotched (left) and notched (middle) beams loaded in three point bending and the tension of half-depth prisms (right) using the probabilistic discrete mesoscale model, denoted by black empty circles and errorbars. The colored curves show results obtained with the proposed model based on extremes of averaged random fields. The four different values of standard deviation (coefficients of variation) of the local strength random fields, $\delta_{h}$, are highlighted in the boxes.

and standard deviation as

$$
\mu_{\mathrm{p}, \infty}=\mu_{\mathrm{d}}, \quad \delta_{\mathrm{p}, \infty}=\sqrt{\delta_{\mathrm{d}}^{2} \delta_{h}^{2}+\mu_{\mathrm{d}}^{2} \delta_{h}^{2}+\delta_{\mathrm{d}}^{2}}
$$

The match with random discrete simulations is absolute. Here, we recall the explanation from Sec. 2 of [8] that for $\ell_{\rho} \uparrow \infty$ the structural strength is simply proportional to variable $h$. This is a consequence of the selected alternative of scaling tensile strength, $f_{\mathrm{t}}$, and fracture energy, $G_{\mathrm{t}}$, in such a manner that the mesoscale Irwin/Hillerborg characteristic length is kept constant.

Let us now the study the behavior for intermediate values of autocorrelation length. The monotonic increase in the standard deviation $\delta_{\mathrm{p}}\left(\ell_{\rho}, \delta_{h}\right)$ of the peak load with an increasing autocorrelation length $\ell_{\rho}$ is affected by the averaging volume $T_{1} T_{2} T_{3}$ via the variance reduction function. Having a good match with the standard deviation confirms the selection/identification of the RVE volume. Since $T_{3}$ must be the beam width $b$, the only free parameters of the model are the proportion $T_{2} / T_{1}$ and the chain length $L_{1}$. The pair denoted $T_{1}$ and $L_{1}$ influences the dependence of the average strength $\delta_{\mathrm{p}}\left(\ell_{\rho}, \delta_{h}\right)$ on the autocorrelation length and the random 
field variance. When the autocorrelation length $\ell_{\rho}$ is roughly of the order of the averaging length $T_{1}$, the average strength $\mu_{\mathrm{p}}$ undergoes a noticeable drop, which becomes pronounced with increasing random field variance. The $T_{1} / \ell_{\rho}$ ratio is small enough to limit the averaging effect and yet large enough to activate the weakest-link effect. In this way, the probabilistic length scale $\ell_{\rho}$ and the deterministic length scale $T_{1}$ interact.

A deeper understanding of the mechanisms leading to the drop in average strength for certain combinations of the parameters of the random field can be achieved by analyzing the presented analytical model. It is a result of a combination of several factors, among which the derivative of the effective strength profile, $\delta_{\dot{H}}$, plays a role. Indeed, the increase in $\delta_{\dot{H}}$ is detrimental to the average structural strength in two different ways:

- The mean upcrossing rate of the averaged random field

- The effective chain length $L_{1}$ is proportional to the gradient: $L_{1} \propto \delta_{\dot{H}}$ (in bent beams).

The length of the virtual chain is, however, not the only factor influencing the mean strength. Any increase in $\ell_{\rho}$ also leads to an increase in the standard deviation of the averaged random field, which is detrimental to the strength of the chain. The interplay of these effects leads to the relatively complicated dependence of the minimum average chain strength on the two parameters of the random field: $\ell_{\rho}$ and $\delta_{h}$. For the studied range of standard deviations $\delta_{h}$, the critical autocorrelation lengths vary between 20 and $35 \mathrm{~mm}$ (unnotched beams) and $6-10 \mathrm{~mm}$ (unnotched beams); see the left and middle columns in Fig. 7.

While the strength troughs are very pronounced in the unnotched beams, in notched simulations the average peak load is found to be only very weakly sensitive to the spatial variability in material parameters. The reason is that the stress concentration is so severe that the crack is forced to propagate from one specific location (the notch tip) and the spatial variability in material parameters is not sufficient to change the location of dissipative processes. In other words, the weakest-link effect is strongly reduced. However, the standard deviation of the peak load decreases with the decrease in the autocorrelation length due to the averaging of the fluctuations within the RVE, which was found to be independent of the applied random field in [8].

\section{CONCLUSIONS}

This paper promotes a recently published analytical model that predicts statistics for the random strength of beams made of quasibrittle materials in which strength is assumed to vary according to a random field, in particular a homogeneous isotropic random field with Gaussian distribution and an arbitrary separable autocorrelation function. These strength statistics can also be obtained via costly discrete mesoscale simulation with additional local strength variability modelled by a random field. We argue that if data obtained from the random discrete mesoscale model [8] are used, the erratic patterns of local stresses in a concrete body may be replaced by locally averaged (effective) stresses for the purpose of the overall strength analysis. Failure becomes governed by a nonlocal criterion. If the local stress average exceeds a critical threshold at any location, the system (structure) is assumed to attain its peak load. Here the critical threshold is the effective strength obtained as the local average of strengths with a certain volume of the heterogeneous material.

The proposed approach relaxes two assumptions of the classical Weibull theory: (i) the consideration of effective strength reflects the progressive loss in material integrity and the associated stress redistribution within a representative volume element (RVE) that takes place prior to reaching the peak load and, (ii) the consideration of spatial correlation departs from the Weibullian assumption of the independence of strengths at various locations. The RVE window encloses the smallest material volume whose failure may trigger the failure of the whole struc- 
ture. The dependence of its strength on the point variance and on the autocorrelation length of the random field is elucidated. The analysis provides insight into the interaction between (i) the probabilistic length scale (introduced via the autocorrelation length of the random strength field) and (ii) the deterministic length scale (expressed via the dimension of the averaging RVE).

The model is shown to agree well with results obtained for three point bend specimens with and without a notch that were analyzed using the random discrete mesoscale model. The uniaxial tension of prisms is shown to exhibit more complicated behavior for which the model's assumptions do not hold. The presented model illustrates the transformation of a threedimensional structure into a chain of RVEs. In the case of notched beams, the possibility of sampling a crack location randomly is quite limited by the stress concentration, and thus the weakest-link principle is suppressed to a high extent. The strength of notched beams is only modified in terms of its variance, but the distribution of beam strength is almost exactly proportional to the strength of a single RVE. In unnotched beams, the weakest-link principle modifies the distribution from that of a single RVE to the extreme value type.

The model has the deterministic/energetic features embodied by considering the redistribution within an RVE (three lengths). Given this simplification and the knowledge of the spatial variability of local material strength, the entire size effect (the dependence of the mean and standard deviation of structural strength on size) can be explained from a pure statistical viewpoint.

\section{ACKNOWLEDGMENT}

The author acknowledges financial support provided by the Czech Science Foundation via project no. GC19-06684J.

\section{REFERENCES}

[1] Zdeněk P. Bažant and Sze-Dai Pang. Activation energy based extreme value statistics and size effect in brittle and quasibrittle fracture. Journal of the Mechanics and Physics of Solids, 55(1):91-131, 2007.

[2] Zdeněk P. Bažant and Jaime Planas. Fracture and Size Effect in Concrete and Other Quasibrittle Materials. CRC Press, Boca Raton and London, 1998.

[3] Z. P. Bažant and Zhengzhi Li. Modulus of rupture: Size effect due to fracture initiation in boundary layer. Journal of Structural Engineering, 121(4):739-746, 11995.

[4] Zdenek P. Bažant. Can multiscale-multiphysics methods predict softening damage and structural failure? International Journal for Multiscale Computational Engineering, 8(1):61-67, 2010.

[5] Gianluca Cusatis and Luigi Cedolin. Two-scale study of concrete fracturing behavior. Engineering Fracture Mechanics, 74(1-2):3-17, 2007.

[6] Gianluca Cusatis, Daniele Pelessone, and Andrea Mencarelli. Lattice discrete particle model (LDPM) for failure behavior of concrete. I: Theory. Cement \& Concrete Composites, 33(9):881-890, 2011.

[7] Henry Ellis Daniels. The statistical theory of the strength of bundles of threads. I. Proceedings of the Royal Society of London. Series A. Mathematical and Physical Sciences, 183(995):405-435, 1945. 
[8] Jan Eliáš and Miroslav Vořechovský. Fracture in random quasibrittle media: I. Discrete meso-scale simulations of load capacity and fracture process zone. Engineering Fracture Mechanics, 235:107160, 2020.

[9] Jan Eliáš, Miroslav Vořechovský, Jan Skoček, and Zdeněk P. Bažant. Stochastic discrete meso-scale simulations of concrete fracture: comparison to experimental data. Engineering Fracture Mechanics, 135:1-16, 2015.

[10] R. A. Fisher and L. H. C. Tippett. Limiting forms of the frequency distribution of the largest and smallest member of a sample. Mathematical Proceedings of the Cambridge Philosophical Society, 24(2):180-190, 1928.

[11] D. G. Harlow, R. L. Smith, and H. M. Taylor. Lower tail analysis of the distribution of the strength of load-sharing systems. Journal of Applied Probability, 20(2):358-367, 1983.

[12] R. Hill. Elastic properties of reinforced solids: Some theoretical principles. Journal of the Mechanics and Physics of Solids, 11(5):357-372, 1963.

[13] H. Moussaddy, D. Therriault, and M. Lévesque. Assessment of existing and introduction of a new and robust efficient definition of the representative volume element. International Journal of Solids and Structures, 50(24):3817-3828, 2013.

[14] M. Ostoja-Starzewski. Microstructural randomness versus Representative Volume Element in thermomechanics. Journal of Applied Mechanics, 69(1):25-35, 062001.

[15] Martin Ostoja-Starzewski. Material spatial randomness: From statistical to representative volume element. Probabilistic Engineering Mechanics, 21(2):112 - 132, 2006.

[16] M. Stroeven, H. Askes, and L.J. Sluys. Numerical determination of representative volumes for granular materials. Computer Methods in Applied Mechanics and Engineering, 193(30):3221-3238, 2004. Computational Failure Mechanics.

[17] Miroslav Vořechovský. Incorporation of statistical length scale into Weibull strength theory for composites. Composite Structures, 92(9):2027-2034, 2010.

[18] Miroslav Vořechovský and Jan Eliáš. Fracture in random quasibrittle media: II. Analytical model based on extremes of averaging process. Engineering Fracture Mechanics, 235:107155, 2020.

[19] Miroslav Vořechovský and Václav Sadílek. Computational modeling of size effects in concrete specimens under uniaxial tension. International Journal of Fracture, 154(1-2):2749, 2008.

[20] W. Weibull. The phenomenon of rupture in solids. Royal Swedish Institute of Engineering Research (Ingenioersvetenskaps Akad. Handl.), Stockholm, 153:1-55, 1939.

[21] W. Weibull. A Statistical Theory of the Strength of Materials, volume (Handlingar Nr.) 151 of Royal Swedish Institute of Engineering Research (Ingeniörsvetenskapsakademiens). Generalstabens litografiska anstalts förlag, Stockholm, 1939.

[22] Zhifeng Xu and Jia-Liang Le. A first passage based model for probabilistic fracture of polycrystalline silicon mems structures. Journal of the Mechanics and Physics of Solids, 99:225-241, 2017. 